\title{
Ocorrência de doença periodontal, cárie e perda dentária em tabagistas pacientes de uma clínica-escola de Odontologia no sul do estado de Minas Gerais: estudo caso-controle
}

\author{
Occurrence of periodontal disease, caries, and tooth loss in smoker \\ patients of a Dental Clinic-School in the south region of the state \\ of Minas Gerais: case control study
}

\author{
Letizia Monteiro de Barros* \\ Marília Gomes Gouvêa** \\ Ingrid Sabrina Moura Reis** \\ Jacqueline de Carvalho Bueris* \\ Laís de Lima Pereira** \\ Roberta Bessa Veloso Silva**
}

\section{Resumo}

Objetivo: comparar a ocorrência de doença periodontal, cárie e perda dentária em tabagistas de ambos os gêneros, adultos, admitidos para tratamento odontológico integrado em uma escola de odontologia, comparados a um grupo-controle não tabagista. Métodos: foram avaliados exames clínicos dentários e periodontais em prontuários de 31 pacientes fumantes e de 46 não fumantes, quanto à ocorrência de: sangramento e/ ou supuração gengival; perda de suporte periodontal por bolsa ou retração gengival; hipermobilidade dentária; dentes cariados, restaurados e dentes perdidos. Para comparar as médias entre os grupos, foi utilizado $o$ teste $t$ de Student $(p \leq 0,005)$ e a correlação entre as variáveis foi obtida por análise multivariada. Resultados: os tabagistas relataram fumar, em média, 11,32 \pm 8,599 cigarros/dia, sendo que $54,83 \%$ deles fumavam mais de 10 cigarros/dia. Observou-se que as variáveis: número de dentes com perda de inserção, bolsa periodontal, retração gengival, hipermobilidade, sangramento/supuração, perda média de inserção (em $\mathrm{mm}$ ) e idade estão altamente correlacionadas e são diretamente proporcionais. $O$ número de dentes hígidos foi inversamente proporcional à idade e o índice de placa correlacionou-se com o número de dentes cariados, perdidos e com cálculo. Entretanto, não foi observada diferença estatisticamente significativa entre os grupos para nenhum parâmetro, exceto quanto à perda dentária, com os fumantes apresentando, em média, mais dentes perdidos do que os não fumantes $(p=0,0171)$. Conclusão: o tabagismo não influenciou significativamente na ocorrência de cárie ou de doença periodontal na população estudada, porém os fumantes têm maior perda dentária do que os não fumantes, e deveriam ser aconselhados a abandonar o tabaco.

Palavras-chave: Cárie. Doença periodontal. Perda dentária. Tabagismo.

\section{Introdução}

Há muitas evidências clínicas e epidemiológicas dos efeitos adversos do tabaco sobre a saúde bucal. O hábito de fumar está relacionado com aumento da incidência e severidade das doenças periodontais, da taxa de dentes perdidos, além de alterações dentárias e da mucosa bucal ${ }^{1}$.

Doutora em Biologia Bucodental, área de Microbiologia Oral da FOP-Unicamp. Professora de Periodontia e Clínica Odontológica Integrada da Universidade José do Rosário Vellano - Unifenas, Alfenas, MG, Brasil.

Acadêmicas do Curso de Odontologia da Universidade José do Rosário Vellano - Unifenas, Alfenas, MG, Brasil.

** Doutora em Estatística e Experimentação Agropecuária da Universidade Federal de Lavras - Ufla. Professora de Bioestatística da Universidade José do Rosário Vellano - Unifenas, Alfenas, MG, Brasil. 
O fumo é um fator de risco fortemente associado à prevalência e à severidade da periodontite ${ }^{2-4}$, possivelmente em uma relação dose dependente ${ }^{3,5,6}$. Em um estudo com 164 pares de gêmeos, na Suécia, o grau de perda óssea alveolar e o número de dentes perdidos foram maiores nos gêmeos altamente expostos ao tabaco, em relação ao seu par menos exposto $^{7}$. No entanto, ainda não foram claramente estabelecidos os mecanismos pelos quais isso ocorre, podendo haver associação com maior número de periodontopatógenos na microbiota, alterações na composição da saliva e distúrbios na resposta do hospedeiro, observados em tabagistas ${ }^{4,8,9}$.

O tabagismo é considerado fator ambiental relevante também para retração ou recessão gengival, que ocorre pela migração apical da gengiva, resultando em exposição radicular ${ }^{3,10-12}$. Uma alta prevalência de retração foi encontrada em uma população urbana brasileira, cujos indicadores de risco mais significativos incluíam o aumento da idade e a exposição ao tabaco ${ }^{11}$. Maior risco de atividade de cárie $^{4,13,14}$ e edentulismo ${ }^{13,15}$ são outras condições relacionadas ao fumo.

Em relação aos sinais clínicos de gengivite, a maioria dos estudos relata uma menor tendência ao sangramento gengival em sujeitos fumantes do que em não fumantes ${ }^{3,9,16-18}$, possivelmente, devido à alteração da resposta inflamatória gengival ao acúmulo de placa, observada em fumantes ${ }^{19}$.

No entanto, há também achados conflitantes, por exemplo, alguns estudos não observaram uma diminuição do sangramento gengival relacionado ao uso do tabaco ${ }^{17,20,21}$. Ramseier et al. ${ }^{17}$ (2015), analisando dados retrospectivos de sangramento à sondagem em 445 pacientes admitidos em um serviço de terapia periodontal de suporte na Suíça, observaram um aumento médio estatisticamente significativo entre sangramento gengival e severidade e progressão da doença periodontal; entretanto, isso ocorreu independentemente do tabagismo. Outros estudos avaliando alterações como retração gengi$\mathrm{val}^{22}$, perda dentária e índice $\mathrm{CPOD}^{23}$ também não encontraram correlação com o tabaco.

Em suma, o hábito de fumar tem uma gama de efeitos deletérios na saúde bucal, porém, muitos aspectos dessa relação ainda não são bem compreendidos.

O objetivo do presente estudo foi avaliar, por meio de dados disponíveis em prontuários, as condições de saúde bucal de pacientes tabagistas e não tabagistas em relação à ocorrência de doença periodontal, cárie e perda dentária.

\section{Sujeitos e método}

Esta pesquisa foi aprovada pelo Comitê de Ética em Pesquisa da Universidade José do Rosário Vellano (Unifenas) (CAAE: 51931615.4.0000.5143), e um termo de consentimento livre e esclarecido foi assi- nado pelos voluntários, autorizando o uso de seus dados, de acordo com as diretrizes e normas éticas.

Foram avaliados prontuários do ano de 2015 de 31 pacientes tabagistas e de 46 não tabagistas (grupos caso e de controle, respectivamente), sendo excluídos aqueles com relato de diabetes, comprometimento imune, uso contínuo de corticoides ou antibióticos. Os prontuários estão arquivados no curso de Odontologia da Unifenas, localizada na cidade de Alfenas, MG.

Foram incluídos na pesquisa apenas os prontuários cujos exames estivessem conferidos e assinados pelos professores responsáveis, especialistas na área, sendo os exames periodontais conferidos por um único professor, especialista em periodontia. Foram examinados todos os dentes presentes, incluindo os terceiros molares. Para avaliar as condições dentárias, foi observado o número de dentes restaurados, cariados, perdidos e dentes hígidos, sem cáries ou restaurações.

As sondagens periodontais eram feitas utilizando sonda milimetrada manual Goldman Fox/ Williams (Hu-Friedy), tomando-se seis medidas por dente, sendo três do lado vestibular e três medidas do lado lingual/palatino; foram sondadas, assim, as faces mesial, distal e a face livre correspondente de cada dente (foram consideradas as profundidades de sondagem $\geq 3 \mathrm{~mm}$ ).

As retrações gengivais foram mensuradas nas faces vestibulares e linguais/palatinas, equivalentes à distância do colo anatômico do dente até a margem gengival (foram consideradas as retrações $\geq 1 \mathrm{~mm}$ ). Para avaliar a perda média de inserção periodontal foram somadas as medidas referentes às bolsas periodontais com as de retrações gengivais $(\mathrm{mm})$, por paciente, e o total dividido pelo número de dentes presentes.

Foi observado também o índice de placa inicial ${ }^{24}$; o número de dentes com hipermobilidade; sangramento/supuração à sondagem e cálculo supra ou subgengival. Nenhum indivíduo havia recebido tratamento periodontal prévio, porém, anteriormente à sondagem periodontal e ao exame dentário, cada paciente recebeu, conforme sua necessidade, uma sessão de adequação do meio bucal, que consiste de raspagens supragengivais, instrução de higiene, selamento provisório de cavidades de cárie e atendimentos de urgência, porém, os dentes com cálculo foram anotados antes da raspagem. Esses procedimentos prévios constituem um protocolo de atendimento da clínica integrada, para propiciar condições adequadas para exame dental e sondagem mais acurados.

Para comparar as médias dos fumantes e dos não fumantes, em relação às variáveis analisadas, foi utilizado o teste $\mathrm{t}$ de Student ao nível nominal de $5 \%$ de significância ${ }^{25}$. As correlações entre as variáveis foram obtidas por meio de uma análise multivariada ${ }^{26}$. Todas as análises foram realizadas no software estatístico R CORE TEAM ${ }^{27}$. 


\section{Resultados}

Foram avaliados dados de 31 pacientes tabagistas (22 mulheres e 9 homens), com média de idade de 42,22 \pm 9,196 anos, e de 46 não tabagistas (27 mulheres e 19 homens), com média de idade de $40,02 \pm 8,866$ anos. Os tabagistas relataram fumar, em média, 11,32 $\pm 8,599$ cigarros/dia, sendo que $54,83 \%$ fumavam mais de 10 cigarros/dia.

Quanto às alterações periodontais, o diagnóstico foi desde gengiva sadia, passando por gengivite induzida por placa, até periodontite crônica, com vários graus de severidade; não foi diagnosticado nenhum caso de periodontite agressiva. Com uma análise multivariada dos dados, foi obtido um mapa de variáveis (Figura 1), no qual se observa que as variáveis: número de dentes com perda de inserção; bolsa periodontal; retração gengival; hipermobilidade; sangramento/supuração; perda média de inserção (em mm) e idade estão altamente correlacionadas e são diretamente proporcionais, ou seja, à medida que aumentar $x$ unidades em uma delas, acarretará um aumento em todas as outras.

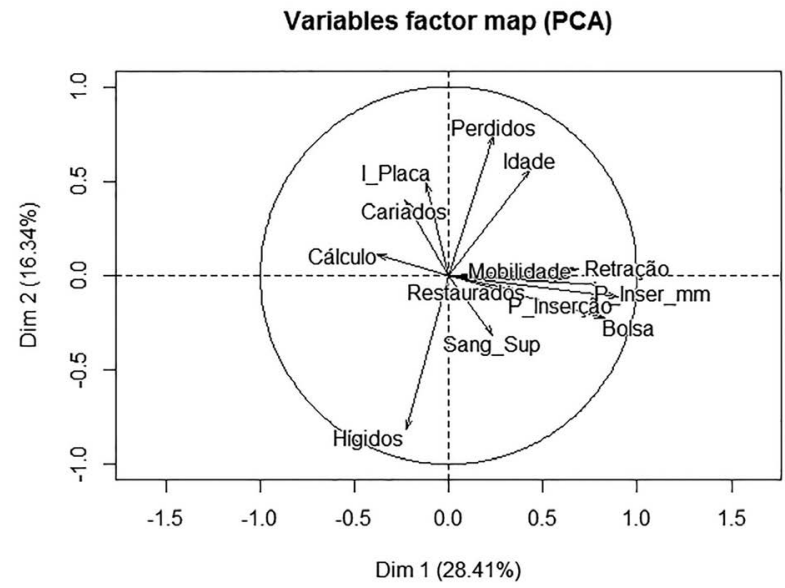

Figura 1 - Mapa de variáveis e a correlação entre as variáveis

Fonte: dos autores.

Destaca-se que, à medida que a idade aumenta, tende a aumentar todas as outras variáveis, exceto o número de dentes hígidos. Pode ser verificado, ainda na Figura 1, que a variável índice de placa está correlacionada com o número de dentes cariados, perdidos e com cálculo.

No mapa de observações (Figura 2), a distribuição da amostra no primeiro quadrante direito está correlacionada com as variáveis que se encontram nos dois quadrantes inferiores do mapa de variáveis, ou seja, número de dentes com perda de inserção, hipermobilidade, sangramento e também número de dentes com restaurações. Entretanto, verificou-se uma distribuição homogênea de fumantes e não fumantes, de forma que não foi possível distingui-los no mapa.

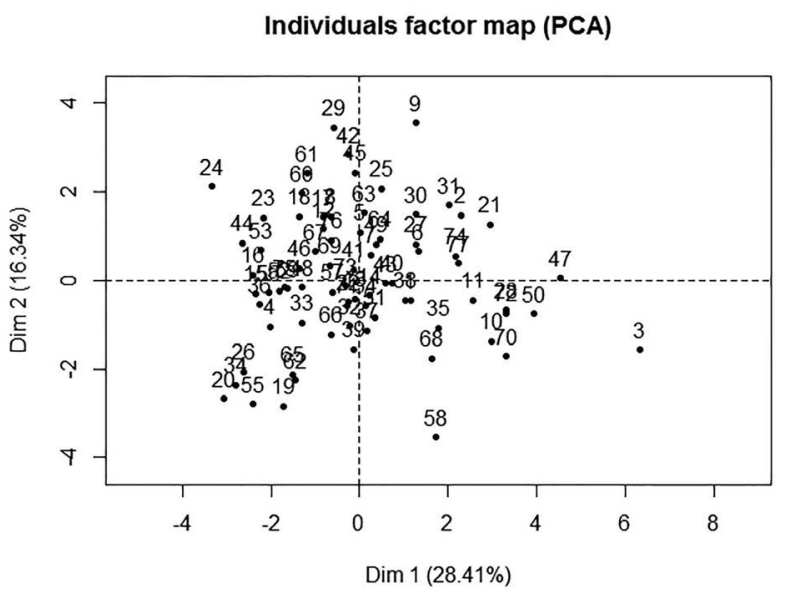

Figura 2 - Mapa de observações e a distribuição dos participantes Fonte: dos autores.

Chama a atenção, no mapa de observações, a maior concentração de participantes correlacionados com as variáveis que se encontram do lado esquerdo (inferior e superior) do mapa de variáveis, ou seja, índice de placa, número de dentes cariados e dentes com cálculo. Porém, também não houve distinção entre os grupos.

As médias entre os grupos, fumantes e não fumantes, também foram calculadas utilizando o teste t de Student ao nível nominal de 5\% de significância (Tabela 1). De acordo com os dados apresentados na Tabela 1, a média de dentes perdidos foi estatisticamente diferente entre os dois grupos, com o dos tabagistas apresentando uma maior perda dentária em relação ao de controle. Para as demais variáveis, no entanto, as médias foram consideradas iguais.

Tabela 1 - Relação entre o hábito de fumar (fumantes e não fumantes), as variáveis de interesse e seus respectivos p-valores

\begin{tabular}{l|c|c|c}
\hline \multirow{2}{*}{\multicolumn{1}{c|}{ Variáveis }} & \multicolumn{2}{c|}{ Médias } & \multirow{2}{*}{ P-valor } \\
\cline { 2 - 3 } & Fumantes & $\begin{array}{c}\text { Não } \\
\text { fumantes }\end{array}$ & \\
\hline Idade & 42,22 & 40,02 & $0,2961 \mathrm{~ns}$ \\
Sangramento e/ou** & 15,84 & 17,87 & $0,2556 \mathrm{~ns}$ \\
supuração & 5,93 & 5,48 & $0,7448 \mathrm{~ns}$ \\
Cálculo** & 4,52 & 4,24 & $0,8438 \mathrm{~ns}$ \\
Bolsa** & 6,45 & 4,41 & $0,0655 \mathrm{~ns}$ \\
Retração** & 8,90 & 7,72 & $0,4815 \mathrm{~ns}$ \\
Perda de inserção** & 5,99 & 6,26 & $0,7752 \mathrm{~ns}$ \\
Perda de inserção & & & \\
(média em mm) & 0,97 & 0,76 & $0,7132 \mathrm{~ns}$ \\
Mobilidade dentária** & 6,48 & 4,09 & $0,0171^{*}$ \\
Dentes perdidos** & 6,32 & 5,24 & $0,2921 \mathrm{~ns}$ \\
Dentes cariados** & 7,48 & 8,65 & $0,2961 \mathrm{~ns}$ \\
Dentes restaurados** & 8,22 & 10,00 & $0,1861 \mathrm{~ns}$ \\
Dentes hígidos** & 51,71 & 56,09 & $0,3014 \mathrm{~ns}$ \\
\hline Índice de placa & & & \\
\hline
\end{tabular}

Fonte: dos autores.

* Significativo ao nível nominal de $5 \%(p<0,05)$; ns = não significativo; ${ }^{*}$ variáveis que se referem ao número de dentes (em média). 


\section{Discussão}

Evidências de estudos transversais e casos-controles, em várias populações, têm demonstrado que adultos fumantes têm uma probabilidade maior de ter periodontite do que os não fumantes, além de uma resposta diminuída à terapia periodontal ${ }^{8}$.

Há muitos relatos de que os fumantes têm menor tendência ao sangramento gengival do que os não fumantes ${ }^{3,9,16-18}$; outros, no entanto, encontraram resultados divergentes, ou seja, um índice de sangramento gengival maior em fumantes ${ }^{21}$, ou, então, não observaram nenhuma diferença consistente quanto ao status gengival em relação ao uso do tabaco ${ }^{18,20,28}$.

No presente estudo, os fumantes apresentaram uma média de 15,84 dentes com sangramento e/ou supuração, enquanto que não fumantes apresentaram 17,87 dentes com essas mesmas características. Assim, essa diferença não foi significativa estatisticamente ( $p=0,2556)$, e o mesmo ocorreu em relação ao índice de placa (Tabela 1). Deve ser considerado que esses pacientes receberam, previamente ao exame periodontal, uma sessão de adequação do meio bucal e, assim, exceto quanto ao cálculo dental, todos os demais parâmetros referem-se à ocorrência residual, avaliada após, aproximadamente, uma semana desse procedimento.

Uma maior média do índice de higiene oral em fumantes nigerianos foi encontrada ${ }^{21}$, sendo esse fato relacionado ao manchamento e a rugosidades causadas pelo tabaco na superfície dos dentes, incrementando o acúmulo de placa. Entretanto, os fumantes não necessariamente têm pior higiene em comparação aos não fumantes. Vários estudos ${ }^{3,13,16,28}$ não encontraram diferença estatisticamente significativa entre tabagistas e não tabagistas em relação ao acúmulo de placa e cálculo. Uma pesquisa, com o objetivo de avaliar a formação de placa em nove fumantes e dez não fumantes do gênero masculino ${ }^{18}$, concluiu que os fumantes apresentaram menores porcentagens de sítios com placa subgengival e de inflamação gengival que os não fumantes.

A variação do sangramento gengival, em grande proporção, pode ter outras causas, além do acúmulo de placa. Trombelli e Farina ${ }^{29}$ (2013), em uma revisão da literatura, observaram uma variabilidade individual significativa na resposta inflamatória gengival, independente de diferenças qualitativas e quantitativas do acúmulo de placa, ou seja, em condições quase idênticas em relação ao desafio bacteriano. Sugere-se que tal resposta possa ser uma característica individual, possivelmente de ordem genética, podendo ser modificada por fatores sistêmicos, inerentes ao hospedeiro, ou relacionada a influências ambientais. Em uma pesquisa com amostra populacional composta por universitários tabagistas e não tabagistas, foram encontradas maiores proporções de patógenos periodontais em fumantes, além de um padrão diferente da resposta inflama- tória entre os grupos durante o curso de 28 dias de gengivite experimental ${ }^{9}$.

No presente estudo, não foram encontradas diferenças significativas em nenhum parâmetro periodontal avaliado. Assim, foi estatisticamente semelhante, entre fumantes e não fumantes, o número de dentes com cálculo, profundidade de bolsa, perda de inserção e mobilidade dentária. De modo interessante, nem mesmo a ocorrência de retração gengival foi significativa, apesar de os fumantes apresentarem, em média, maior número de dentes com retração gengival em relação aos não fumantes, com 6,45 versus 4,41 dentes com retração, respectivamente $(\mathrm{p}=0,0655)$.

Uma correlação dose dependente entre o hábito de fumar e os indicadores da gravidade da doença periodontal, incluindo perda óssea alveolar, número de dentes perdidos, perda de inserção e profundidade média de sondagem, foi relatada ${ }^{6}$. Uma correlação entre o número de cigarros fumados e o risco de ter periodontite também foi encontrada, sendo observado que indivíduos que fumam nove ou menos cigarros por dia têm seis vezes menos chance de desenvolver periodontite do que aqueles que fumam 31 ou mais cigarros por dia.

Do mesmo modo, outra pesquisa demonstrou que os fumantes apresentavam uma probabilidade 2,7 vezes maior de ter doença periodontal estabelecida, ocorrendo uma relação dose-dependente entre o consumo de cigarros e a probabilidade de ter doença periodontal avançada. Isso foi mais evidente após dez anos do uso do tabaco e, principalmente, em homens. Os autores concluíram que o tabagismo é um fator de risco fortemente associado à periodontite e que seu efeito depende do número de cigarros fumados e da duração do hábito ${ }^{3}$.

Martinez-Canut et al. ${ }^{5}$ (1995) também demonstraram um efeito dose dependente do tabagismo sobre o nível de inserção à sondagem, não sendo encontrada diferença significativa entre fumantes que consumiam menos de 10 cigarros por dia e não fumantes, enquanto para o consumo de 11 a 20 cigarros/dia, ou mais de 20 , os fumantes foram significativamente mais afetados, sugerindo, portanto, que o tabaco aumenta a severidade da doença periodontal, mas que esse efeito é clinicamente evidente a partir do consumo de uma determinada quantidade de tabaco. Entretanto, outro estudo ${ }^{30}$ não encontrou relação dose dependente entre doença periodontal e o número de cigarros fumados por dia ou a duração do hábito de fumar.

No presente trabalho, infelizmente, não estava disponível nos prontuários, para a maioria dos pacientes, o tempo de uso do tabaco, o que traz uma limitação na análise dos resultados. Os indivíduos relataram fumar, em média, aproximadamente onze cigarros por dia; portanto, é possível que a semelhança das condições de saúde bucal entre fumantes e não fumantes seja em razão da intensidade do tabagismo.

Outros fatores, contudo, são considerados importantes no estabelecimento da periodontite crôni- 
ca $^{31}$, como patógenos específicos e a própria genética do indivíduo, portanto, há diferenças individuais quanto à influência do tabagismo na doença periodontal. Vários indicadores relevantes de risco periodontal também incluem: stress, osteopenia com deficiência de estrógeno, diferenças entre os gêneros, idade e fatores hereditários ${ }^{2}$.

A retração gengival é uma manifestação comum da doença periodontal, estando também associada a diversos fatores de risco. Um estudo epidemiológico dessa condição foi conduzido em uma amostra representativa da população brasileira urbana para análise dos vários indicadores de risco, no qual os autores observaram, utilizando um modelo multivariado, que o tabagismo e a presença de cálculo supragengival foram os fatores mais significativamente associados com retrações gengivais localizadas e generalizadas ${ }^{10}$. Esses resultados concordam com os de outro estudo ${ }^{12}$, em que foi observado que os tabagistas apresentam maior risco de retração que os não tabagistas.

Contudo, avaliando o desenvolvimento de retração gengival, dentro de um período de 6 meses, em 61 jovens adultos, fumantes e não fumantes, dos quais 30 fumavam, no mínimo, 20 cigarros por dia, Müller et al. ${ }^{22}$ (2002) observaram que $50 \%$ dos indivíduos apresentaram retração gengival em um ou mais sítios, porém, não houve diferença significativa na prevalência de retração entre os grupos. Retrações consideradas severas pelos autores, ou seja, além de $2 \mathrm{~mm}$, afetaram aproximadamente $23 \%$ dos não fumantes, mas apenas $7 \%$ dos fumantes. Os autores concluíram que seus dados não suportavam a hipótese de que os fumantes estão em risco aumentado de desenvolvimento de retração gengival.

$\mathrm{Na}$ presente pesquisa foi observada, também por análise multivariada (Figura 1), uma alta correlação entre as variáveis: idade; número de dentes com perda de inserção; bolsa periodontal; retração gengival; hipermobilidade; sangramento/supuração; perda média de inserção (em mm), e uma relação inversamente proporcional da idade com o número de dentes hígidos.

Estudos epidemiológicos têm demonstrado uma associação do aumento da prevalência e severidade da doença periodontal com o aumento da idade ${ }^{32}$, com o processo inflamatório gengival iniciando-se em fases precoces e progredindo com a idade, até atingir o periodonto de sustentação ${ }^{33}$.

Também quanto à cárie dentária, há resultados controversos na literatura, com relatos que associam o tabagismo com maior risco de cárie e de perda dentária $^{1,13,14}$ e maior índice $\mathrm{CPOD}^{34}$. Entretanto, outro estudo ${ }^{23}$, investigando a prevalência de cárie e o hábito de fumar, em 505 recrutas de 19 anos de idade, na Croácia, não encontrou diferença estatística quanto ao índice CPOD nem quanto aos dentes perdidos entre fumantes e não fumantes. Porém, a idade relativamente baixa dos indivíduos desse estudo poderia interferir na comparação com outras pesquisas.
No presente estudo também não se observou associação significativa do tabagismo com o número de dentes cariados, restaurados ou hígidos. Porém, o número de dentes perdidos foi significativamente maior nos fumantes do que nos não fumantes $(p=0,0171)$. Mai et al. ${ }^{35}$ (2013), em um estudo para verificar possíveis associações entre o tabagismo e as causas da perda dentária, em mulheres pós-menopausa, encontraram que o hábito de fumar pode ser o principal fator da perda dentária devido à doença periodontal, mas parece ser menos importante nas perdas por cárie.

Embora com alguns resultados conflitantes, é evidente na literatura o efeito danoso do tabagismo sobre a saúde bucal, e os cirurgiões-dentistas têm a responsabilidade de orientar seus pacientes fumantes sobre a importância da cessação do hábito. Pesquisas abordando associação entre os vários fatores de risco, incluindo intensidade do consumo do tabaco, gênero, idade e genética, poderiam ser úteis para determinar quais populações têm maior chance de sofrer condições adversas bucais em relação ao tabagismo.

\section{Conclusão}

Dentro dos limites deste estudo, pode-se concluir que o tabagismo não influenciou significativamente a ocorrência de cárie ou de doença periodontal, porém, os fumantes têm maior perda dentária do que não fumantes, e deveriam ser aconselhados a abandonar o tabaco. Uma condição dentária e periodontal ruim foi encontrada, em geral, nessa população.

\section{Abstract}

Objective: to compare the occurrence of periodontal disease, caries, and tooth loss in adult smokers of both genders admitted for integrated dental treatment in a dental school, to a non-smoker control group. Methods: dental and periodontal clinical exams were evaluated in medical records of 31 smokers and 46 non-smokers regarding the occurrence of bleeding and/or gingival suppuration, loss of periodontal support by pocket or gingival retraction, tooth hypermobility, and decayed, missing, and filled teeth. Student $t$ test $(p \leq 0.005)$ was used to compare the averages between groups, and the correlation between variables was obtained by multivariate analysis. Results: smoker patients reported smoking an average of $11.32 \pm 8.599$ cigarettes/day and $54.83 \%$ of them smoked more than 10 cigarettes/day. It was observed that variables of number of teeth with insertion loss, periodontal pocket, gingival retraction, hypermobility, bleeding/suppuration, average insertion loss (in $\mathrm{mm}$ ), and age are highly correlated and directly proportional. The number of healthy teeth was inversely proportional to age, and plaque index was correlated to the number of decayed and lost teeth and dental calculus. However, no statistically significant difference was observed between groups for any parameter, except for tooth loss, with smokers having an average of more lost 
teeth than non-smokers ( $p=0.0171)$. Conclusion: smoking did not significantly influence the occurrence of caries or periodontal disease in the population studied, but smokers present higher tooth loss than non-smokers, and they should be advised to quit smoking.

Keywords: Caries. Periodontal disease. Tooth loss. Smoking.

\section{Referências}

1. Rad M, Kakoie S, Brojeni FN, Pourdamghan N. Effect of long-term smoking on whole-mouth salivary flow rate and oral health. J Dent Res Dent Clin Dent Prospect 2010; 4(4):110-14.

2. Genco RJ. Current view of risk factors for periodontal diseases. J Periodontol 1996;67(10 Suppl):1041-9.

3. Calsina G, Ramón JM, Echeverría JJ. Effects of smoking on periodontal tissues. J Clin Periodontol 2002; 29(8):771-6.

4. Reibel J. Tobacco and oral diseases. Update on the Evidence, with Recommendations. Med Princ Pract 2003;12(suppl. 1):22-32.

5. Martinez-Canut P, Lorca A, Magan R. Smoking and periodontal disease severity. J Clin Periodontol 1995;22:743-49.

6. Bernardes VS, Ferres MO, Lopes Junior W. O tabagismo e as doenças periodontais. FOL 2013;23(1):37-45.

7. Bergström J, Floderus-Myrhed B. Co-twin control study of the relashionship between smoking and some periodontal disease factors. Community Dent Oral Epidemiol 1983;11:113-6.

8. Johnson GK, Hill M. Cigarette smoking and the periodontal patient. J Periodontol 2004;75(2):196-209.

9. Peruzzo DC, Gimenes JH, Taiete T, Casarin RC, Feres M, Sallum EA, et al. Impact of smoking on experimental gingivitis. A clinical, microbiological and immunological prospective study. J Periodontal Res 2016;51(6):800-11.

10. Susin C, Haas AN, Oppermann RV, Haugejorden O, Albandar JM. Gingival Recession: Epidemiology and Risk Indicators in a Representative Urban Brazilian Population. J Periodontol 2004;75(10):1377-86.

11. Rios FS, Costa RSA, Moura MS, Jardim JJ, Maltz M, Haas AN. Estimates and multivariable risk assessment of gingival recession in the population of adults from Porto Alegre, Brazil. J Clin Periodontol 2014;41:1098-107.

12. Chambrone L, Chambrone D, Pustiglioni FE, Chambrone LA, Lima LA. The influence of tobacco smoking on the outcomes achieved by root-coverage procedures: a systematic review. J Am Dent Assoc 2009;140(3):294-306.

13. Axelsson P, Paulander J, Lindhe J. Relationship between smoking and dental status in 35-, 50-, 65-, and 75-year-old individuals. J Clin Periodontol 1998; 25(4):297-305.

14. Gaio EJ, Rösing, CK. Cessação do hábito de fumar: responsabilidade da odontologia. Clín Int J Braz Dent 2013; 9(1):106-9.

15. Peltzer K, Hewlett S, Yawson AE, Moynihan P, Preet R, Wu F, et al. Prevalence of Loss of All Teeth (Edentulism) and Associated Factors in Older Adults in China, Ghana, India, Mexico, Russia and South Africa. Int J Environ Res Public Health 2014;11:11308-24.

16. Chen X, Wolff L, Aeppli D, Guo Z, Luan W-M, Baelum V, et al. Cigarette smoking, salivary/gingival crevicular fluid cotinine and periodontal status. A 10-year longitudinal study. J Clin Periodontol 2001;28:331-9.

17. Ramseier CA, Mirra D, Schütz C, Sculean A, Lang NP, Walter $\mathrm{C}$, et al. Bleeding on probing as it relates to smoking status in patients enrolled in supportive periodontal therapy for at least 5 years. J Clin Periodontol 2015;42(2):150-9.
18. Branco P, Weidlich P, Oppermann RV, Rösing CK. Early supra- and subgingival plaque formation in experimental gingivitis in smokers and never-smokers. Oral Health Prev Dent 2015;13(1):13-20.

19. Bergström J, Preber H. The influence of cigarette smoking on the development of experimental gingivitis. J Periodontal Res 1986;21:668-76.

20. Bergström J, Eliasson S, Dock J. 10-Year Prospective Study of Tobacco Smoking and Periodontal Health. J Periodontol 2000;71:1338-47.

21. Arowojolu MO, Fawole OI, Dosumu EB, Opeodu OI. A comparative study of the oral hygiene status of smokers and non-smokers in Ibadan, Oyo state. Niger Med J 2013; 54(4):240-3.

22. Müller HP, Stadermann S, Heinecke A. Gingival recession in smokers and non-smokers with minimal periodontal disease. J Clin Periodontol 2002; 29:129-36.

23. Badel T, Pavicin IS, Carek AJ, Segović S. Dental caries experience and tobacco use in 19-year-old Croatian army recruits. Coll Antropol 2014;38(2):671-5.

24. O'Leary TJ, Drake RB, Naylor JE. The plaque control record. J periodontol 1972;43(1):38.

25. Ferreira DF. Estatística básica. Lavras: Ufla; 2009.

26. Ferreira DF. Estatística multivariada. Lavras: Ufla; 2011.

27. R Development Core Team 2016. R: A language and environment for statistical computing. R Foundation for Statistical Computing, Vienna, Austria. ISBN 3-900051-07-0. [citado em 2016]. Disponível em URL: http://www.R-project.org.

28. Bastiaan RJ, Waite IM. Effects of tobacco smoking on plaque development and gingivitis. J Periodontol 1978; 49(9):480-2.

29. Trombelli L, Farina R. A review of factors influencing the incidence and severity of plaque-induced gingivitis. Minerva Stomatol 2013;62(6):207-34.

30. Ismail AI, Burt BA. Eklundand periodontal disease in the United States. J Am Dent Assoc 1983;106(5):617-21.

31. Polk DE, Wang X, Feingold E, Shaffer JR, Weeks DE, Weyant RJ, et al. Effects of Smoking and Genotype on the PSR Index of Periodontal Disease in Adults Aged 18-49. Int J Environ Res Public Health 2012; 9(1):2839-50.

32. Machion L, Freitas PM, Cesar Neto JB, Nogueira Filho GR, Nociti Jr. FH. A influência do sexo e da idade na prevalência de bolsas periodontais. Pesq Odont Bras 2000;14(1):33-7.

33. Hugson A, Jordan T. Frequency distribution of individuals aged 20-70 years according to severity of periodontal disease. Community Dent Oral Epidemiol 1982;10:187-92.

34. Al-Habashneh R, Al-Omari MA, Taani DQ. Smoking and caries experience in subjects with various form of periodontal diseases from a teaching hospital clinic. Int J Dent Hyg 2009;7(1):55-61.

35. Mai X, Wactawski-Wende J, Rovey KM, LaMonte MJ, Chen C, Tezal M, et al. Associations between smoking and tooth loss according to reason for tooth loss: the osteoperio study. J Am Dent Assoc 2013;144(3):252-65.

\section{Endereço para correspondência:}

Letizia Monteiro de Barros

Rua Feliciano Libânio da Silveira, no 350,

Bairro Jardim Aeroporto

37130-826 Alfenas - MG

Fone: (35) 98824-6316/ (35) 3299-3158

E-mail: letizia.barros@unifenas.br

Recebido: 16/12/2016. Aceito: 22 /03/2017. 